Asian J Agric \& Biol. 2020;8(1):92-97. DOI: 10.35495/ajab.2019.04.171

\title{
$\mathrm{AJAB}$
}

Short Communication

\section{Effects of cassava starch supplementation on behavioural characteristics and oxidative status in Drosophila melanogaster}

Olarewaju M. Oluba1 ${ }^{*}$, Motunrayo M. Ayodele ${ }^{1}$, Abigail G. Adeyonu ${ }^{2}$, Ayoola J. Shoyombo², Olayinka O. Alabi², Oghenerobor B. Akpor ${ }^{3}$

${ }^{1}$ Department of Biochemistry, Food Safety and Toxicology Research Unit, College of Pure \& Applied Sciences, Landmark University, P.M.B. 1001, Omu-Aran, Kwara State, Nigeria

${ }^{2}$ Department of Agricultural Sciences, Landmark University, P. M. B. 1001, Omu-Aran, Kwara State, Nigeria

${ }^{3}$ Department of Microbiology, Landmark University, P. M. B. 1001, Omu-Aran, Kwara State, Nigeria

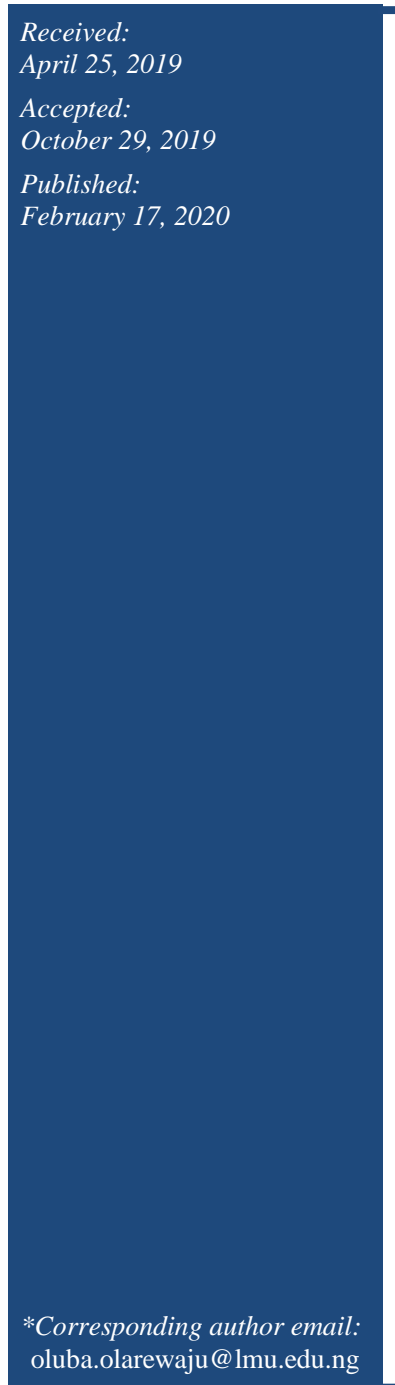

\section{Abstract}

Studies have revealed that adaptive life-history behaviour and reproductive capacity of an organism are influenced by the amount and quality of nutrients. Furthermore, it has been established that the balance between energy production and utilization is crucial to the animal's survival and reproduction vis-a-vis the organism's ability to adjust their developmental, physiological or behavioural response to environmental conditions. In the present study an attempt was made to investigate the effect of cassava starch supplementation on chill-coma recovery, reproductive capacity and some metabolicinduced changes in oxidative status of Drosophila melanogaster, which was raised on a normal fly diet supplemented with 500, 750, 1000, 1250, 1500 and $2000 \mathrm{mg} / \mathrm{mL}$ starch extracted from three cassava cultivars (TMS 92/0326, TMS 30572 and OmuAran local sweet cassava). Data generated from this study showed that flies consuming starch-supplemented diet have a higher level of cold resistance, an increased survival rate as well as enhanced egg to adult viability. However, flies raised on starchsupplemented diet showed a significantly higher concentration of thiobarbituric acid reactive substances (TBARS) with a compensatory significant increase in activities of antioxidant defense enzymes, and nitric oxide scavenging capacity compared to flies raised on the normal fly diet. In conclusion, data obtained from this study revealed that supplementation to the D. melanogaster diet with cassava starch enhances the organism's survival rate, and adaptive response to cold stress, with concomitant improvement in antioxidant status.

Keywords: Drosophila melanogaster diet, Starch supplementation, Adaptive behaviour, Oxidative stress

\section{How to cite this:}

Oluba OM, Ayodele MM, Adeyonu AG, Shoyombo AJ, Alabi OO and Akpor OB, 2020. Effects of cassava starch supplementation on behavioural characteristics and oxidative status in Drosophila melanogaster. Asian J. Agric. Biol. 8(1):92-97. DOI: 10.35495/ajab.2019.04.171

This is an Open Access article distributed under the terms of the Creative Commons Attribution 3.0 License. (https://creativecommons.org/licenses/by/3.0), which permits unrestricted use, distribution, and reproduction in any medium, provided the original work is properly cited. 


\section{Introduction}

For many omnivorous and herbivorous species, including humans and animals, carbohydrates are important dietary components. Most cellular reactions are driven by energy derived from available carbohydrate. Consequently, most organisms tend to adjust their body physiology in relation to the available dietary carbohydrate source. Physiological effects of dietary carbohydrates depend on their type and dosage, as well as on the physiological state of an organism (Lushchak et al., 2013). A low intake of carbohydrates limits the energy reserve of an organism and can slow growth and regeneration, thereby altering survival and safety (Wheeler and Pi-Sunyer, 2008). Over the years, dietary modifications have been employed as a viable strategy to elucidate the mechanisms underlying certain adaptive traits in Drosophila melanogaster including reproductive, developmental as well as behavioural traits (Piper, 2017; Trajković et al., 2017). Furthermore, it has been established that the interplay between energy input and utilization is crucial to the determination of an organism's survival and reproduction and to a great extent the capacity of organism to adjust its developmental, physiological or behavioural response to varying environmental conditions.

Dietary adjustment through experimentation has been reported to impact significantly on the capacity of an organism to efficiently allocate their energy resources (Cruz-Neto and Bozinovic, 2004; Nuñez-Villegas et al., 2014). Reports on the effect of dietary carbohydrates on body physiology have been limited to humans and rodent models. More so, a majority of such studies were carried out using pre-established disease models of diabetes, obesity, or hypertension. Thus, studies focused on the effects of dietary carbohydrate on animal survival and reproductive capacities are scanty.

The use of Drosophila melanogaster as a suitable model for nutritional studies has been widely reported in literature. Although several studies have explored the effect of diet on life span, reproduction and adaptive behaviour of fruit flies (Lee et al., 2008; Catterson et al., 2010; Yurgel et al., 2015; Abrat et al., 2018; Staats et al., 2018), none has specifically focused on the inclusion of dietary starch. Therefore, this study sought to evaluate the effects of starch supplementation on behavioural, reproductive capacity and changes in oxidative status in $D$. melanogaster.

\section{Material and Methods}

\section{Fly stock and husbandry}

D. melanogaster flies were obtained from the Drosophila Laboratory, University of Ibadan, Nigeria and subsequently maintained for more than ten generations on simple culture medium containing agar-agar, dried yeast, corn flour, nipargin and plain water at $25{ }^{\circ} \mathrm{C}$, under a 12:12 light:dark cycle, and $60 \%-70 \%$ relative humidity.

\section{Cassava samples}

Freshly harvested tubers of two different cassava varieties; TMS92/0326 and TMS 30572 were obtained from the International Institute of Tropical Agriculture (IITA), Ibadan, Oyo State Nigeria. Tubers from locally cultivated sweet cassava (designated local) cultivar were also obtained in Omu-Aran, Kwara State, Nigeria. The different cassava tubers were transported to Landmark University, Omu-Aran Kwara State Nigeria (where the study was carried out) in clean jute sacs.

\section{Cassava starch preparation}

Cassava starch was prepared from tubers of the three different cassava cultivars using the method described by Sriroth et al. (2000). The cassava tubers were washed, peeled, washed again, grated and sieved with a substantial quantity of water (10 times, the volume of the mash) using a muslin cloth. The sieved solution was allowed to settle (sediment) after which the supernatant was decanted. The starch obtained was dewatered and then dried in a hot air oven (Gallenkamp Co. Ltd London England) at $70{ }^{\circ} \mathrm{C}$ for 4 $\mathrm{h}$. The dried starch was then milled into powder using a milling machine and sieved (sieve aperture, $0.4 \mathrm{~mm}$ ) to obtain fine flour. The starch sample for each cassava cultivar was stored in an airtight container at $4{ }^{\circ} \mathrm{C}$ for further analysis.

\section{Preparation of normal diet}

The media bottles were washed and rinsed with tap water and then sterilized in hot air oven at $60{ }^{\circ} \mathrm{C}$ for 5 $6 \mathrm{~h}$. Distilled water $(700 \mathrm{~mL})$ was poured into 1000 $\mathrm{mL}$ beaker and allowed to boil on a hot plate. On boiling, $10 \mathrm{~g}$ of yeast and $7.9 \mathrm{~g}$ of agar was added slowly with incessant stirring for 10 minutes. Corn flour $(52 \mathrm{~g})$ dissolved in $750 \mathrm{~mL}$ distilled water was added to the mixture on a hot plate and stirred for 15 min after which it was allowed to cool to $60{ }^{\circ} \mathrm{C}$. 
Nipargin (5 g) was added as an antibacterial and antifungal agent. The viscosity of the media was checked before pouring into the media bottles.

\section{Experimental diet}

The experimental diet was prepared by adding starch (5\%) from the three cassava varieties to the normal Drosophila diet as described above. The diets were prepared according to the method of Abrat (2015).

\section{Survival rate}

Fly survival rate was estimated according to the method of Chhabra et al. (2013). Briefly, newly enclosed flies (25 males and 25 females/vial) were collected under light anesthesia and randomly assigned into the respective treatment groups. Each treatment was carried out in triplicates. Flies in each treatment were passed every other day and the number of dead flies were counted daily and used to evaluate their survival rate.

\section{Negative geotaxis}

The locomotor performance of the flies fed with control and experimental diet was investigated using the negative geotaxis test, according to Coulom and Birman (2004), with minor modifications. Ten flies from each treatment group were rendered motionless under anesthesia with ice after which they were placed separately in a vertical glass column $(15 \mathrm{~cm} \times 1.5 \mathrm{~cm})$. After $30 \mathrm{~min}$ of recovery from anesthesia, the flies were gently tapped to the bottom of the column and allowed to climb through the column while monitoring the time lapse to climb to the $8 \mathrm{~cm}$ mark. The tests were repeated three times for each fly at 1-min interval.

\section{Chill coma recovery}

The fly ability to withstand and recover from chillinduced coma was monitored following the procedures described by Nilson et al. (2006).

\section{Biochemical assays}

Glucose concentration was determined according to the glucose oxidase method of Trinder (1969). Lipid peroxidation was estimated based on the procedure of Buege and Aust (1978). Superoxide dismutase (SOD) and catalase activities were determined according to McCord and Fridovich (1973) and Aebi (1974) respectively. Nitric oxide scavenging activity was estimated according to the method of Marcocci et al. (1994).

\section{Statistical analysis}

The results are presented as the mean \pm standard error of mean (SEM) of triplicate biological assays. The comparison of mean values was by One-way Analysis of Variance (ANOVA) followed by Turkey's Multiple Comparison using SPSS version 17 while $p<0.05$ was considered significant.

\section{Results}

Flies fed TMS 30572 and local cassava starch supplemented diet had higher chance of survival compared to flies fed TMS 92/0326 cassava starch and normal diets (Fig. 1).

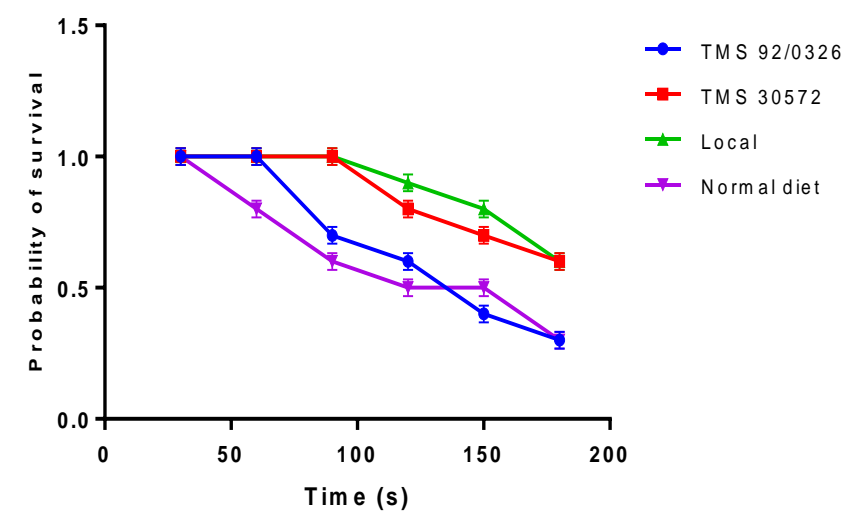

Figure-1: Survival curve for chill-coma recovery in Drosophila melanogaster flies fed cassava starch supplemented diet

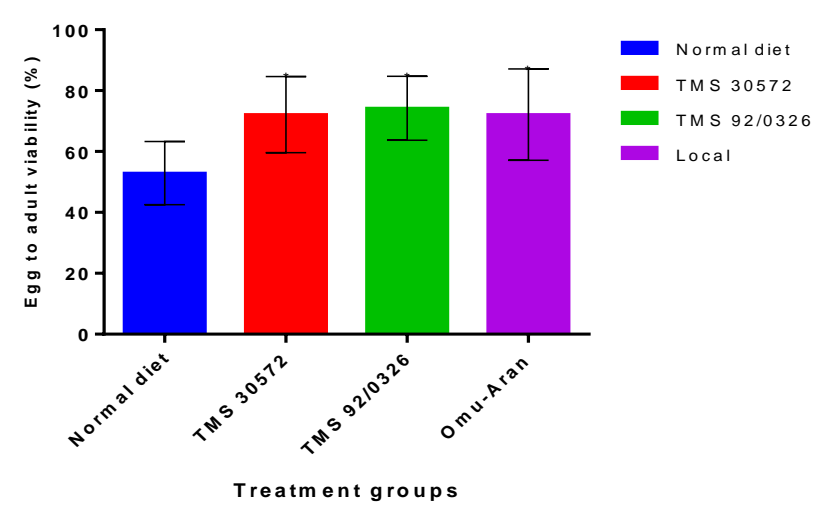

Figure-2: Egg to adult viability of flies developing on cassava starch supplemented diet

Flies fed diets supplemented with the respective cassava starch had greater percentage egg to adult viability compared to flies fed normal fly diet. No 
significant $(p>0.05)$ difference was observed in percentage egg to adult viability between flies fed cassava starch-supplemented diets (Figure 2).

Glucose concentration was significantly higher $(p<$ 0.05) in flies fed starch-supplemented diet compared to normal diet. Flies fed cassava starch obtained from the local cassava cultivar had significantly $(p<0.05)$ higher glucose concentration compared to flies fed starch obtained from TMS 92/0326 and TMS 30572 cassava cultivars (Figure 3 ).

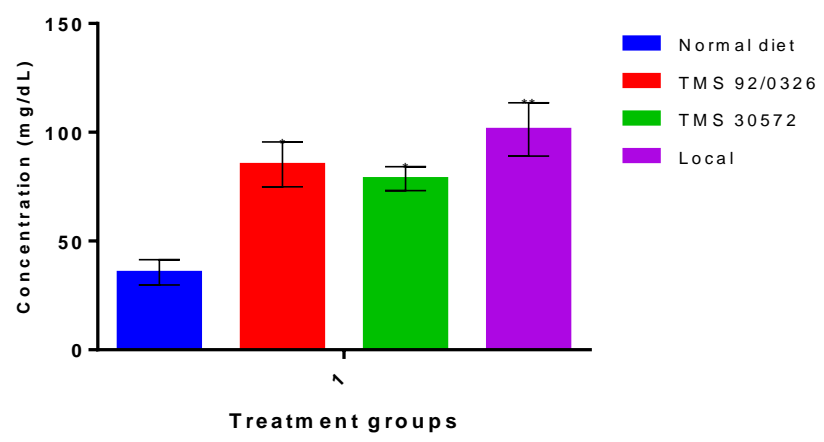

Figure-3: Glucose concentration level in Drosophila melanogaster flies kept on cassava starch supplemented diet

Lipid peroxide level in flies fed starch-supplemented diet and normal diet was not significantly altered. Similarly, flies fed cassava starch-supplemented diets showed no significant $(p>0.05)$ difference in the MDA concentrations (Figure 4).

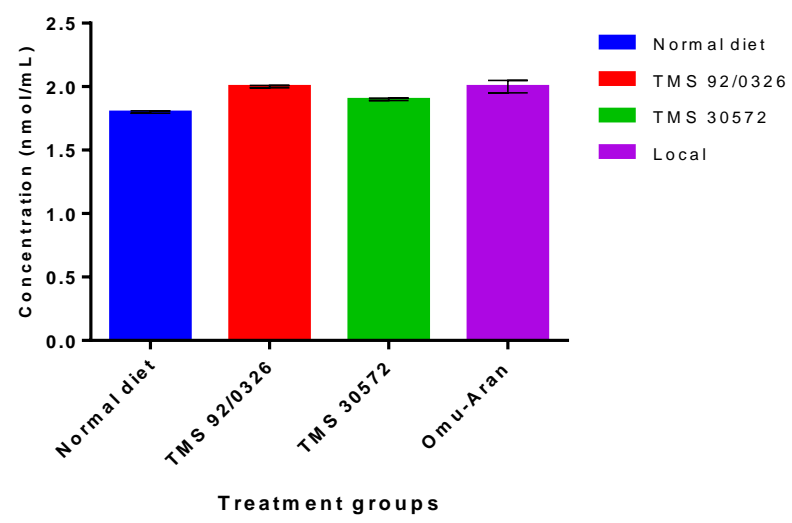

Figure-4: Lipid peroxidation level in Drosophila melanogaster flies kept on cassava starch supplemented diet

Superoxide dismutase (SOD) and catalase (CAT) activities were significantly $(\mathrm{p}<0.05)$ higher in flies fed starch supplemented diet compared to flies fed normal diet. In addition, the percentage nitric oxide scavenging activity was significantly $(\mathrm{p}<0.05)$ higher in flies fed starch-supplemented diet compared to flies fed normal diet. However, there was no significant ( $p$ $>0.05$ ) difference in terms of SOD and CAT activities as well as percentage nitric oxide scavenging activity between flies fed cassava starch-supplemented diets (Figure 5).

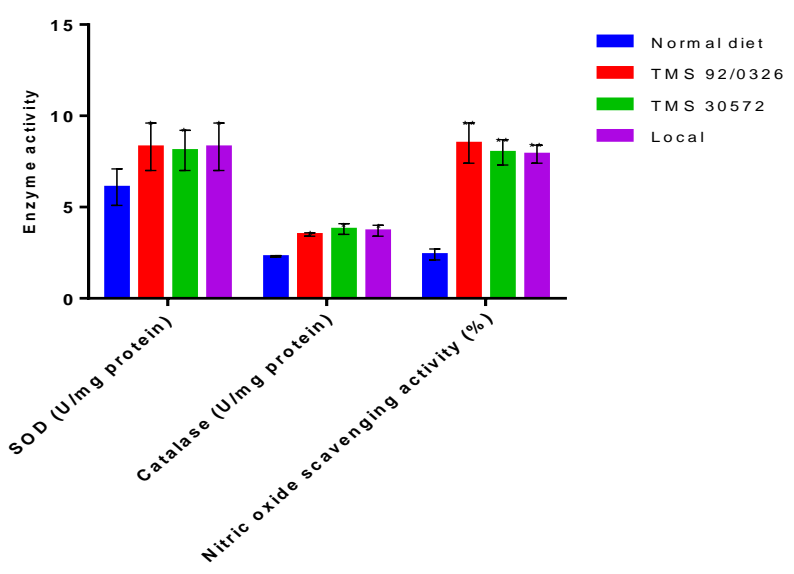

Figure-5: Oxidative status of Drosophila melanogaster flies kept on cassava starch supplemented diet

\section{Discussion}

The survival rate of flies to a great extent depends on temperature, diet and other environmental conditions (Driver and Lamb, 1980). In this research flies whose diet was supplemented with cassava starch from TMS92/0326 cultivar showed the highest survival rate.

The ability of an organism to recover from chillinduced coma is greatly influenced by both genetic and environmental factors (Macmillan and Sinclair, 2011). From previous research, D. melanogaster has been shown to manifest improved resistance to cold when fed calorie-laden and protein-enriched diet (Chen et al., 1993). Findings from this study showed that flies fed the diet supplemented with cassava starch had a higher recovery rate from chill-induced coma compared to those fed the normal fly diet. The balance between energy intake and expenditure has been reported to contribute both to the survival and reproductive success of animals (Metcalfe and Monaghan, 2003; Salmon et al., 2001). This balance depends on the interplay between energy intake, 
digestion and allocation of acquired energy on one hand, and various functions such as maintenance, growth and reproduction on the other hand (Baker and Thummel, 2007). Experimental modifications of animal diets have played a key role in the study of how organisms adjust their energy allocation (Chown and Nicolson, 2004; Cruz-Neto and Bozinovic, 2004).

Cassava starch supplementation as demonstrated in this study thus provided an extra energy source which could be channeled to various functions such as maintenance, growth and reproduction (Shintani et al., 2018). This observation could explain the higher survival rate and greater tendency to withstand cold stress. It is also pertinent to note that increased calorie loading may translate to increased metabolic rate and thus higher tendency for a generation of free radical as a result of an incomplete reduction of molecular oxygen to water. Thus, the observed increase in lipid peroxidation in flies grown on starch supplemented diet could be as a result of the high calorie-induced metabolic abnormality bringing about an increased generation of free radicals.

SOD and catalase are two of the key enzymes that mop up free radicals from a cellular system. The activity of these enzymes is induced following an increased generation of reactive oxygen species (ROS). The increased carbohydrate loading as a consequence of starch supplementation in this study may have led to an increased generation of ROS as evident in a higher lipid peroxidation rate in flies fed diets containing starch supplement, and ultimately leading to the induced synthesis of these enzymes (SOD and catalase) (Di Naso et al., 2012; Furukawa et al., 2017). Thus, the observed increase in the activities of antioxidant enzymes in D. melanogaster flies fed on cassava starch-supplemented diet could have been a resultant effect of the elevated ROS in these flies. Increased oxidative stress as a result of a high carbohydrate/calorie diet is an established feature of metabolic syndromes associated with obesity, because oxidative stress may favour cell damage due to a generation of pro-inflammatory substances such as nitric oxide (Lushchak et al., 2011). However, results from this study showed increased nitric oxide scavenging potential to flies fed cassava supplemented diet. Obesity caused by derangement in carbohydrate metabolism and/or increased lipid deposition in adipose tissue has been reported to initiate cellular oxidative stress, culminating in chronic inflammation mediated through an enhanced generation of nitric oxide by adipose tissue (Di Naso et al., 2012).

\section{Conclusion}

Data obtained from this study revealed that supplementation of the normal D. melanogaster diet with cassava starch enhanced the organism's survival rate, and adaptive response to cold stress, with concomitant upregulation of antioxidant enzyme activity.

\section{Disclaimer: None.}

Conflict of Interest: None. Source of Funding: None.

\section{References}

Abrat O, 2015. Influence of amylose starch on development and lifespan of fruit fly Drosophila melanogaster. J. Vasyl Stefanyk Precarpathian Natl. Univ. 2(1):100-6.

Abrat OB, Storey JM, Storey KB and Lushchak VI, 2018. High amylose starch consumption induces obesity in Drosophila melanogaster and metformin partially prevents accumulation of storage lipids and shortens lifespan of the insects. Comp. Biochem. Physiol. Part A: Mol. Integ. Physiol. 215:55-62.

Aebi H, 1974. Catalase. In: Methods of enzymatic analysis. pp. 673-684. Academic Press, USA.

Baker KD and Thummel CS, 2007. Diabetic larvae and obese flies - emerging studies of metabolism in Drosophila. Cell Metab. 6(4): 257-266.

Buege JA and Aust SD, 1978. Microsomal lipid peroxidation. In: Methods in enzymology. 52: 302310. Academic Press, USA.

Catterson JH, Knowles-Barley S, James K, Heck MM, Harmar AJ and Hartley PS, 2010. Dietary modulation of Drosophila sleep-wake behaviour. PLoS One. 5(8): e12062.

Chen Z, Jiang JC, Lin ZG, Lee WR, Baker ME and Chang SH, 1993. Site-specific mutagenesis of Drosophila alcohol dehydrogenase: evidence for involvement of tyrosine-152 and lysine-156 in catalysis. Biochem. 32(13): 3342-3346.

Chhabra R, Kolli S and Bauer JH, 2013. Organically grown food provides health benefits to Drosophila melanogaster. PLoS One. 28(1): e52988.

Chown SL and Nicolson S, 2004. Insect physiological ecology: mechanisms and patterns. Oxford University Press.

Coulom H and Birman S, 2004. Chronic exposure to rotenone models sporadic Parkinson's disease in Drosophila melanogaster. J. Neurosci. 24(48): 10993-10998. 
Cruz-Neto AP and Bozinovic F, 2004. The relationship between diet quality and basal metabolic rate in endotherms: insights from intraspecific analysis. Physiol. Biochem. Zool. 77: 877-889.

Di Naso FC, Rodrigues G, Dias AS, Porawski M, Fillmann $H$ and Marroni NP, 2012. Hepatic nitrosative stress in experimental diabetes. J. Diabet. Complicat. 26(5): 378-381.

Driver CJI and Lamb MJ, 1980. Metabolic changes in ageing Drosophila melanogaster. Exp. Gerontol. 15(3): 167-175.

Furukawa S, Fujita T, Shimabukuro M, Iwaki M, Yamada Y, Nakajima Y, Nakayama O, Makishima M, Matsuda M and Shimomura I, 2017. Increased oxidative stress in obesity and its impact on metabolic syndrome. J. Clin. Invest. 114(12): 1752-1761.

Lee KP, Simpson SJ, Clissold FJ, Brooks R, Ballard JWO, Taylor PW, Soran N and Raubenheimer D, 2008. Lifespan and reproduction in Drosophila: new insights from nutritional geometry. Proc. Natl. Acad. Sci. 105(7): 2498-2503.

Lushchak OV, Gospodaryov DV, Rovenko BM, Yurkevych IS, Perkhulyn NV and Lushchak VI, 2013. Specific dietary carbohydrates differentially influence the life span and fecundity of Drosophila melanogaster. J. Gerontol. Series A: Biomed. Sci. Med. Sci. 69(1):3-12.

Lushchak V, Rovenko BM, Gospodaryov DV and Lushchak VI, 2011. Drosophila melanogaster larvae fed by glucose and fructose demonstrate difference in oxidative stress markers and antioxidant enzymes of adult flies. Comp. Biochem. Physiol. Part A: Mol. Integ. Physiol. 160(1): 27-34.

MacMillan HA and Sinclair BJ, 2011. Mechanisms underlying insect chill-coma. J. Insect Physiol. 57(1): 12-20.

Marcocci L, Maguire JJ, Droylefaix MT and Packer L, 1994. The nitric oxide-scavenging properties of Ginkgo biloba extract EGb 761. Biochem. Biophys. Res. Comm. 201(2):748-55.

McCord JM and Fridovich I, 1973. Production of $\mathrm{O}_{2}$ in photolyzed water demonstrated through the use of superoxide dismutase. Photochem. Photobiol. 17(2):115-21.

Metcalfe NB and Monaghan P, 2003. Growth versus lifespan: perspectives from evolutionary ecology. Exp. Gerontol. 38(9): 935-940.

Nilson TL, Sinclair BJ and Roberts SP, 2006. The effects of carbon dioxide anesthesia and anoxia on rapid cold-hardening and chill coma recovery in Drosophila melanogaster. J. Insect Physiol. 52(10): 1027-1033.

Nuñez-Villegas M, Bozinovic F and Sabat P, 2014.
Interplay between group size, huddling behavior and basal metabolism: an experimental approach in the social degu. J. Exp. Biol. 217(6): 997-1002.

Piper MD, 2017. Using artificial diets to understand the nutritional physiology of Drosophila melanogaster. Curr. Opin. Insect Sci. 23:104-11.

Salmon AB, Marx DB and Harshman LG, 2001. A cost of reproduction in Drosophila melanogaster: stress susceptibility. Evolut. 55(8): 1600-1608.

Shintani H, Shintani T, Ashida H and Sato M, 2018 Calorie restriction mimetics: upstream-type compounds for modulating glucose metabolism. Nutrients. 10(12):1821.

Sriroth K, Chollakup R, Chotineeranat S, Piyachomkwan $\mathrm{K}$ and Oates CG, 2000. Processing of cassava waste for improved biomass utilization. Bioresour. Technol. 71(1):63-9.

Staats S, Lüersen K, Wagner AE and Rimbach G, 2018. Drosophila melanogaster as a versatile model organism in food and nutrition research. J. Agric. Food Chem. 66(15):3737-53.

Trajković J, Vujić V, Miličić D, Gojgić-Cvijović G, Pavković-Lučić S and Savić T, 2017. Fitness traits of Drosophila melanogaster (Diptera: Drosophilidae) after long-term laboratory rearing on different diets. Eur. J. Entomol. 114(1):222-9.

Trinder P, 1969. Determination of glucose in blood using glucose oxidase with an alternative oxygen acceptor. Ann. Clin. Biochem. 6(1):24-7.

Wheeler ML and Pi-Sunyer FX, 2008. Carbohydrate issues: type and amount. J. Am. Diet Assoc. 108(4):S34-9.

Yurgel ME, Masek P, DiAngelo J and Keene AC, 2015. Genetic dissection of sleep-metabolism interactions in the fruit fly. J. Comp. Physiol. A. 201(9): 869-877.

\section{Contribution of Authors}

Oluba OM: Designed the experiment and wrote and approved the final manuscript Ayodele MM: Carried out the experiment and approved the final manuscript Adeyonu AG: Carried out the experiment and approved the final manuscript

Shoyombo AJ: Performed data analysis and interpretation

Alabi OO: Performed data analysis and interpretation Akpor OB: Interpreted the data and proof-read the manuscript and approved it 